\title{
DIRICHLET PROBLEMS FOR SINGULAR ELLIPTIC EQUATIONS
}

\author{
CHI YEUNG LO
}

ABSTRACT. Boundary value problems are formulated for the equation

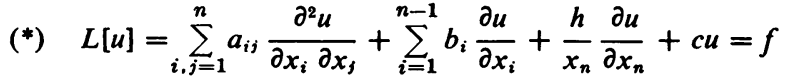

in a bounded domain $G$ in $E_{n}$ with boundary $\partial G=S_{1} \cup S_{2}$ where $S_{1}$ is in $x_{n}=0$ and $S_{2}$ is in $x_{n}>0$. A uniqueness theorem is established for $\left({ }^{*}\right)$ when boundary data is only given on $S_{2}$ for

$$
h\left(x_{1}, \cdots, x_{n-1}, 0\right) \geqq 1 ;
$$

whereas an existence and uniqueness theorem for the Dirichlet problem is proved for $h\left(x_{1}, x_{2}, \cdots, x_{n-1}, 0\right)<1$.

Let $G$ be a bounded domain in a half space $x_{n}>0$ with boundary $\partial G=S_{1} \cup S_{2}$ where $S_{1}$ is contained in the hyperplane $x_{n}=0$ and $S_{2}$ lies entirely in $x_{n}>0$. We consider a class of second order linear partial differential equations

$$
L[u]=\sum_{i, j=1}^{n} a_{i j} \frac{\partial^{2} u}{\partial x_{i} \partial x_{j}}+\sum_{i=1}^{n-1} b_{i} \frac{\partial u}{\partial x_{i}}+\frac{h}{x_{n}} \frac{\partial u}{\partial x_{n}}+c u=f
$$

where the coefficients $a_{i j}(\bar{x}), b_{i}(\bar{x}), h(\bar{x}), c(\bar{x})$ and $f(\bar{x})$ are continuous functions of $\bar{x}=\left(x_{1}, x_{2}, \cdots, x_{n}\right)$ in $\bar{G}$, and $c(\bar{x}) \leqq 0$ in $\bar{G}$. Furthermore, $L[u]$ is uniformly elliptic in $\bar{G}$, i.e. there exists a positive constant $m$ such that

$$
\sum_{i, j=1}^{n} a_{i j}(\bar{x}) \xi_{i} \xi_{j} \geqq m \sum_{i=1}^{n} \xi_{i}^{2}
$$

for all $n$-tuples of real numbers $\left(\xi_{1}, \cdots, \xi_{n}\right)$ and all $\bar{x} \in G$. By normalization, $a_{n n}(\bar{x})=1$.

In this paper we shall formulate some boundary value problems of (1) in $\bar{G}$. Since the coefficient of $\partial u / \partial x_{n}$ goes to infinity as $x_{n} \rightarrow 0$, it is obvious

Received by the editors August 4, 1972.

AMS (MOS) subject classifications (1970). Primary 35J70, 35J 25.

Key words and phrases. Singular elliptic equations, boundary value problem, maximum principle, barrier function, Schauder lemma.

(a) American Mathematical Society 1973 
that the behavior of $h(\bar{x})$ near $x_{n}=0$ plays an important part in our formulation. A well-known example of (1) is given by the generalized axially symmetric potential equation

$$
\sum_{i=1}^{n} \frac{\partial^{2} u}{\partial x_{i}^{2}}+\frac{k}{x_{n}} \frac{\partial u}{\partial x_{n}}=0
$$

where $k$ is a real number.

For $k<1, \mathbf{P}$. Brousse and $\mathrm{H}$. Poncin have shown that the Dirichlet problem can be solved if smooth boundary is admitted [1]. However, for $k \geqq 1$, the Dirichlet problem is in general not solvable. A. Huber has shown that there exists a unique function $u$ in $\bar{G}$, which satisfies (3) and assumes bounded continuous data on $S_{2}$ ([3], [4]). Accordingly, we shall formulate our boundary value problems, based on the assumption whether $h\left(x_{1}, \cdots, x_{n-1}, 0\right)<1$ or $h\left(x_{1}, \cdots, x_{n-1}, 0\right) \geqq 1$.

THEOREM 1. Let $h(\bar{x})$ be a $C^{2}$-function in $\bar{G}$, even in $x_{n}$, and $h\left(x_{1}, x_{2}, \cdots, x_{n-1}, 0\right) \geqq 1$. Then there is at most one solution $u(\bar{x})$ of $(1)$ which is regular in $G$, remains bounded when $x_{n} \rightarrow 0$, and assumes given continuous values $\Phi(\bar{x})$ on the boundary $S_{2}$.

Proof. It is sufficient to prove that any bounded and regular solution $u(\bar{x})$ of $L[u]=0$ which vanishes on $S_{2}$ must vanish identically in $G$. We want to construct a barrier function $w(\bar{x})$ with the following properties: (a) $w(\bar{x})$ is positive in $\bar{G}$, (b) $w(\bar{x})$ converges uniformly to infinity when $x_{n} \rightarrow 0$ and (c) $L[w]<0$.

If such a function $w(\bar{x})$ exists, then, for any $\varepsilon>0$, we form $v(\bar{x})=$ $\varepsilon w(\bar{x})+u(\bar{x})$. Let $P \in G$. Then, from property (b) of $w(\bar{x})$, there exists an $\eta$ such that

$$
v(\bar{x}) \geqq 0 \quad \text { for } 0<x_{n} \leqq \eta .
$$

We require $\eta$ is so small that $P \in G_{\eta}=G \cap D_{\eta}$ where $D_{\eta}=\left\{\tilde{x} \mid x_{n}>\eta\right\}$. This implies $v(\bar{x}) \geqq 0$ on $\partial G_{\eta}$, and, from property (c) of $w(\bar{x}), L[v]<0$. Hence, by maximum principle for elliptic operators [2], $v(\bar{x}) \geqq 0$ in $G_{\eta}$ or $u(P) \geqq-\varepsilon w(P)$. Similarly, by defining $v(\bar{x})=\varepsilon w(\bar{x})-u(\bar{x})$, we obtain $u(P) \leqq \varepsilon w(P)$. Hence $|u(P)| \leqq \varepsilon w(P)$. Since $\varepsilon$ is arbitrary, $u(P)=0$.

The function $w(\bar{x})$ can be defined as

$$
w(\bar{x})=-\ln x_{n}-\left(x_{1}-\alpha\right)^{\beta}+k
$$

where $\alpha$ is chosen such that $x_{1}-\alpha>1$ for all $\bar{x}=\left(x_{1}, \cdots, x_{n}\right) \in G$, whereas $\beta$ and $k$ are positive constants to be determined later.

Then

$$
\begin{aligned}
L[w]= & -a_{11}(\bar{x}) \beta(\beta-1)\left(x_{1}-\alpha\right)^{\beta-2}-b_{1}(\bar{x}) \beta\left(x_{1}-\alpha\right)^{\beta-1} \\
& +(1-h(\bar{x})) / x_{n}^{2}+c(\bar{x}) w(\bar{x}) .
\end{aligned}
$$


Applying Taylor's theorem with remainder to the function $F\left(x_{n}\right)=$ $h\left(\xi_{1}, \cdots, \xi_{n-1}, x_{n}\right)$ where $\xi_{i}, i=1, \cdots, n-1$, are considered as parameters and $\left(\xi_{1}, \cdots \xi_{n}, x_{n}\right) \in G$, we obtain

$$
F\left(x_{n}\right)=F(0)+\left(F^{\prime}(0) / 1 !\right) x_{n}+\left(F^{\prime \prime}\left(\xi_{n}\right) / 2 !\right) x_{n}^{2}
$$

where $0<\xi_{n}<x_{n}$.

Then, from the hypothesis on $h(\bar{x})$, we have

$$
\begin{aligned}
& \frac{1-h\left(\xi_{1}, \cdots, \xi_{n-1}, x_{n}\right)}{x_{n}^{2}} \\
& \quad=\frac{1-h\left(\xi_{1}, \cdots, \xi_{n-1}, 0\right)-h\left(\xi_{1}, \cdots, \xi_{n-1}, 0\right)}{x_{n}^{2}} \\
& \quad<-h_{x_{n} x_{n}}\left(\xi_{1}, \cdots, \xi_{n-1}, \xi_{n}\right) \leqq A
\end{aligned}
$$

where $A$ is chosen to be a positive constant greater than $\max _{\bar{x} \in \bar{G}}\left|h_{x_{n} x_{n}}(\bar{x})\right|$. Moreover,

$$
a_{11}(\bar{x})>m \text { by (2). }
$$

Hence, combining (5), (6) and (7), we have

$$
L[w]<-m \beta(\beta-1)\left(x_{1}-\alpha\right)^{\beta-2}-\beta b_{1}(\bar{x})\left(x_{1}-\alpha\right)^{\beta-1}+A+C(\bar{x}) w(\bar{x}) .
$$

Choose $\beta$ such that $m(\beta-1)>\max (3 B, 2)$ and $\beta(\beta-1)>3 A / m$ where $B=\max _{\bar{x} \in G}\left|b_{1}(\bar{x})\left(x_{1}-\alpha\right)\right|$.

The number $k$ is now chosen such that the equation $w^{\prime}(\bar{x})$ is positive everywhere in $G$. Then,

$$
\begin{aligned}
L[w] & <-m \beta(\beta-1)+\frac{1}{3} m \beta(\beta-1)+A+c(\bar{x}) w(\bar{x}) \\
& <-(2 m \beta / 3)(\beta-1)+A<-m \beta(\beta-1) / 3<0 .
\end{aligned}
$$

In order to show that the Dirichlet problem is solvable when $h\left(x_{1}, \cdots, x_{n-1}, 0\right)<1$, we plan to apply the Schauder theorem on the regular elliptic differential equations.

For $0<\alpha<1$, let $C^{m+\alpha}(\bar{D})$ be the set of functions $u \in C^{m}(\overline{\mathscr{D}})$ whose $m$ th

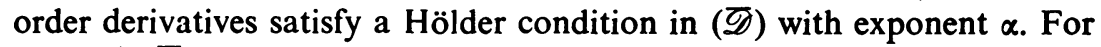
$u \in C^{m+\alpha}(\overline{\mathscr{Q}})$, define

$$
\|u\|_{m+\alpha}^{\mathscr{O}}=\max _{x \in \mathscr{\mathscr { P }}} \sum_{k=0}^{m}\left|\mathscr{D}^{k} u\right|+\max _{P, Q \in \mathscr{D}} \frac{\left|D^{m} u(P)-D^{m} u(\mathcal{O})\right|}{\mid P-\mathcal{O}^{\alpha}} .
$$

Then the Schauder result can be stated as follows. (See [5], [6].) 
Lemma (SChauder). Let $\mathscr{D}$ be a bounded domain with boundary $\partial \mathscr{D}$ of class $C^{2+\alpha}$. Let

$$
\mathscr{L}(u)=\sum_{i, j=1} a_{i j} \frac{\partial^{2} u}{\partial x_{i} \partial x_{j}}+\sum_{i=1}^{n} b_{i} \frac{\partial u}{\partial x_{i}}+c u=f
$$

be a uniformly elliptic differential equation in $\mathscr{D}$. Assume that the coefficients $a_{i j}, b_{i}, c, f$ are in $C^{\alpha}(\mathscr{D})$ satisfying $\left|a_{i j}\right|,\left|b_{i}\right|,|c|,|f| \leqq K, a$ constant and $c \leqq 0$ in $\overline{\mathscr{D}}$.

If $\phi$ is any continuous function on $\partial \mathscr{D}$, there exists a unique solution $u \in C^{2+\alpha}(\mathscr{D}) \cap C^{0}(\mathscr{D})$ of $(8)$ with $u=\phi$ on $\partial \mathscr{D}$.

Accordingly, we formulate the following existence theorem:

THEOREM 2. Let $G$ be a bounded domain in $x_{n}>0$ with boundary $\partial G=$ $S_{1} \cup S_{2}$ of class $C^{2+\alpha}$ where $S_{1}$ is a subset of $x_{n}=0$ and $S_{2}$ in $x_{n}>0$. Let the coefficients $a_{i j}, k_{i}, h, c$ and $f$ be in $C^{\alpha}(\bar{G})$ satisfying $\left|a_{i j}\right|,\left|b_{i}\right|,|h|,|f| \leqq K$, and $C \leqq 0$ in $\bar{G}$.

If $\phi$ is any continuous function on $\partial G$, there exists a unique solution $u \in C^{2+\alpha}(G) \cap C^{0}(\bar{G})$ of $(1)$ with $u=\phi$ on $\partial G$.

Proof. The uniqueness of the solution can be handled easily by Hopf's maximum principle.

Extend the function $\phi$ continuously to the closed domain $\bar{G}$ and denote $M=\max _{\bar{x} \in \bar{G}} \phi(\bar{x})$. Let $\eta>0$, and denote $G_{\eta}=G \cap E\left\{x_{n}>\eta\right\}$. According to Schauder's lemma, there exists a solution $u_{\eta} \in C^{2+\alpha}\left(G_{\eta}\right) \cap C^{0}\left(G_{\eta}\right)$ of (1) with $u_{\eta}=\phi$ on $\partial G_{\eta}$. Extend the function $u_{\eta}$ continuously on the whole domain $\bar{G}$ by defining $u_{\eta}=\phi$ on $\bar{G} \backslash \bar{G}_{\eta}$. In this way we define a family $U$ of functions $\left\{u_{\eta}(\bar{x})\right\}$, in $\bar{G}$ corresponding to all values of $\eta, 0<\eta \leqq$ $\max \left\{x_{n} \mid \bar{x}=\left(x_{1} \cdots x_{n}\right) \in \bar{G}\right\}$.

Let $\eta^{*}$ be a fixed, but arbitrary small number. The family $U$ is uniformly bounded by $M$, and from Schauder's estimate

$$
\|u\|_{\partial+\alpha}^{G_{\eta}} \leqq \tilde{K}\left(\|f\|_{\alpha}^{G_{\eta}}+\|u\|_{0}^{G_{\eta}}\right) \quad([5],[6])
$$

this family is also equicontinuous in $G_{\eta}$. Then, by Arzela's lemma, it is possible to select a sequence $\left\{U_{i}^{(1)}(x)\right\}=\left\{u_{\eta_{i}}(\bar{x})\right\}$ so that $U_{i}^{(1)}$, together with its first and second derivatives, converges uniformly in $G_{\eta}$. The sequence $\left\{U_{i}^{(1)}(x)\right\}$ is chosen in the order that $i>j$ if $\eta_{i}<\eta_{j}$. Hence, for $\eta_{i}<\eta$, all $\left\{U_{i}^{(1)}(\bar{x})\right\}$ are solutions of (1) in $G_{\eta^{*}}$.

For each $j$, let $\eta_{j}^{*}=\eta^{*} / j$. Since $\left\{U_{i}^{(1)}(\bar{x})\right\}$ is also a family of uniform boundedness and equicontinuity in $G_{\eta_{2}^{*}}$, we can select a uniformly convergent subsequence $\left\{U_{i}^{(2)}(\bar{x})\right\}$ in $G_{\eta_{2}^{*}}^{*}$ and so forth.

Now, we choose from the sequences

$$
\begin{gathered}
U_{1}^{(1)}, U_{2}^{(1)}, \cdots, U_{i}^{(1)}, \cdots, \\
U_{1}^{(2)}, U_{2}^{(2)}, \cdots, U_{i}^{(2)}, \cdots
\end{gathered}
$$


the "diagonal" subsequence $\left\{U_{n}^{(n)}(\bar{x})\right\}$ which converges uniformly to a regular solution $u(\bar{x})$ of $(1)$ in $G$ and assumes $\phi(\bar{x})$ on $S_{2}$. We still have to prove that for any $Q \in S_{1}, u(\bar{x})$ is continuous and $u(Q)=\phi(Q)$.

Let $Q=\bar{x}^{0}=\left(x_{1}^{0}, x_{2}^{0}, \cdots, x_{n-1}^{0}, 0\right) \in S_{1}$. We want to construct a barrier function $v(\bar{x})$ with the following properties (a) it is continuous in a sufficiently small neighborhood $w_{Q}=\left\{\bar{x}|| \bar{x}-\bar{x}^{0} \mid<\rho, \quad x_{n} \geqq 0\right\}, \quad$ (b) it vanishes at $Q,(\mathrm{c})$ it is positive in $w_{G} \mid Q$ and (d) $L[v]<-1$.

The function $v(\bar{x})$ can be defined as

where $0<\beta<1$.

$$
v\left(x_{1}, \cdots, x_{n-1}, x_{n}\right)=x_{n}^{\beta}+\sum_{i=1}^{n}\left(x_{i}-x_{i}^{0}\right)^{2}
$$

It is clear that $v(\bar{x})$ satisfies properties (a), (b) and (c). However, we still have to determine the exact value of $\beta$ such that $L[v]<-1$.

By elementary computations,

$$
\begin{aligned}
L[v]= & 2\left(a_{11}(\bar{x})+\cdots+a_{n-1, n-1}(\bar{x})\right)+\beta(\beta-1) x_{n}^{\beta-2} \\
& +2\left[b_{1}(\bar{x})\left(x_{1}-x_{1}^{0}\right)+\cdots+h_{n-1}(\bar{x})\left(x_{n-1}-x_{n-1}^{0}\right)\right] \\
& +\beta h(\bar{x}) x_{n}^{\beta-2}+c(\bar{x}) v(\bar{x}) \\
< & 2(n-1) K+2 \rho(n-1) K+\beta[\beta-1+h(\bar{x})] x_{n}^{\beta-2} .
\end{aligned}
$$

Choose $\beta$ such that $0<\beta<1-h\left(x_{1}, \cdots, x_{n-1}, 0\right)$. Then, by continuity, and for sufficiently small values of $\rho$, we have $L[v]<-1$.

Since $\phi(\bar{x})$ is continuous, for any $\varepsilon>0$, we can find a semicircular neighborhood $w_{Q}^{\prime} \subset w_{Q}$ such that

$$
\phi(Q)-\varepsilon \leqq \phi(P) \leqq \phi(Q)+\varepsilon .
$$

Consider the functions

$$
A(P)=\phi(Q)-\varepsilon-k v(P) \quad \text { and } \quad B(P)=\phi(Q)+\varepsilon+k v(P) .
$$

For a sufficiently large positive number $k$, we have $B(P)>M>A(P)$ on the semicircular part of the boundary $w_{Q}^{\prime}$ and $L[A]>K$ and $L[B]<-K$ everywhere in $w_{Q}^{\prime}$. Choose a nonempty domain $w_{q}=w_{Q}^{\prime} \cap E\left\{x_{n}>q>0\right\}$. On $\partial w_{q}, B(P)>M \geqq U_{n}^{n}(P)$, where $U_{n}^{n}$ is an arbitrary member of the sequence $\left\{U_{n}^{n}\right\}$ and satisfies (1) in $w_{q}$, whereas in $w_{q}$, we have $L\left[B-U_{n}^{n}\right]=$ $L[B]-L\left[U_{n}^{n}\right]<-K+f(\bar{x}) \leqq 0$. Then, by maximum principle, $U_{n}^{n}(P) \leqq B(P)$ in $w_{q}$. Similarly, $U_{n}^{n}(P) \geqq A(P)$ in $w_{q}$. Thus we have $A(P) \leqq U_{n}^{n}(P) \leqq B(P)$. Let $n \rightarrow \infty$;

and then let $P \rightarrow Q$;

$$
A(P) \leqq u(P) \leqq B(P)
$$

$$
f(Q)-\varepsilon \leqq \lim _{P \rightarrow Q} u(P) \leqq f(Q)+\varepsilon
$$


or

$$
\lim _{P \rightarrow Q} u(P)=f(Q) .
$$

REMARK. M. Schechter [5] has established an existence theorem of Dirichlet problem of $L u=f$, similar to Theorem 2 by means of Schauder's lemma, but the conditions on the coefficients of the equation are different from ours and his proof does not make use of the construction of barrier function.

\section{REFERENCES}

1. P. Brousse and $\mathrm{H}$. Poncin, Quelques résultats generaux concernant la détermination de solutions d'équations elliptiques par les conditions aux frontières, Mémoires sur la mécanique des fluides offerts à M. Dimitri P. Riabouchinsky, Publ. Sci. Tech. Ministère de l'Air, Paris, 1954. MR 16, 368.

2. E. Hopf, Elementare Bemerkungen über die Lösungen partieller Differential gleichungen vom elliptischen Typus, S.-B. Preuss. Akad. Wiss. 19 (1927), 147-152.

3. A. Huber, On the uniqueness of generalized axially symmetric potentials, Ann. of Math. (2) 60 (1954), 351-358. MR 16, 258.

4. - Some results on generalized axially symmetric potentials, Proc. Conference on Differential Equations, Univ. of Maryland, College Park, Md., 1956, pp. 147-155. MR 18, 650 .

5. M. Schechter, On the Dirichlet problem for second order elliptic equations with coefficients singular at the boundary, Comm. Pure Appl. Math. 13 (1960), 321-328. MR 22 \#3872.

6. J. Schauder, Über lineare elliptische Differential gleichungur zweiter Ordnung, Math. Z. 38 (1934), 257-282.

7. —_ Numerische Abschätzungen in elliptischen linearen Differential-gleichungen, Studia Math. 5 (1934), 34-42.

Department of Mathematics, Michigan State University, East Lansing, MICHIGAN 48823 